\title{
Research on Detection of Moving Target Based on Hybrid Algorithm Min- ing Method for Sports Video Analysis
}

\author{
Yabin Tan* and Tao Kang \\ Physical Education College of Gannan Normal University, Ganzhou Jiangxi, China
}

\begin{abstract}
With the level of competitive sports to improve sports training, demand is also rising, the last with only coaches intuition training way has been can not meet the demand of competitive sports training stage, in order to adapt to this situation, computer vision technology is increasingly cited the use of physical training, because the accuracy and memory of the machine vision has better than the human eye, to capture a moving target rapidly and effectively, and to record the target motion data, provide more theoretical, data description for the athlete's movement. However, the majority of users want to be able to quickly from the multimedia data to extract the interested content and implicit knowledge (concepts, rules, rules, models and correlation), retrieval and query quickly to take advantage of them, but also can provide the decision support problem solving hierarchy. In order to test the validity of the algorithm, this paper constructs a sports video detection and tracking system on the MATLAB platform, the test results show that, there is no and the methods used to appear the phenomenon of track loss, can achieve the desired effect.
\end{abstract}

Keywords: Motion mining, motion tracking, object segmentation, semantic information in the event of track.

\section{INTRODUCTION}

In recent years, with the development of science and technology, the moving target detection and tracking technology is R mature. But in sports video site, athletes and the background color may quite similar, each block still exist between athletes [1]. Unique sports video poses a great challenge to the target detection and tracking technology. Therefore, the purpose of this paper is to propose motion target detection and tracking algorithm is efficient, and built a simulation system to verify the correctness of the algorithm. Because of the accuracy and memory of the machine vision is better than the human eye, can quickly capture a moving target, and can target motion data record [2]. Through the collection of high level athletes in training and competitions in a large number of video image information, for the information to be effective analysis, to change the previous coaches only by manual observation and experience skill coach is insufficient, can greatly improve the training effect [3].

Moving target detection and tracking system is the intelligent recognition system based on the technology of digital image processing, pattern recognition, computer vision based. This system can be widely used in traffic management, the observation of celestial bodies, biomedical research, traffic statistics and sports fields [4]. In the field of sports video analysis, technical detection and tracking of moving object more play an essential role, followed by realtime detection of athletes in action, you can analyze the motion trajectory, convenient and correct athletes in training or human in the game was unable to detect subtle action differences, so as to improve the training effect and competition results [5]. Therefore, combining the research topic detection and tracking, the target of sports video of the in-depth study and discussion, the other moving object tracking based on the field also has a positive impact, has very important theory and application value [6].

This paper mainly in the detection of sports video tracking is the focus of the study, proposed a novel hybrid algorithm, and the extracted images are simple pretreatment, reduces interference of ingredients for the late key algorithm. In addition, this paper also presents detection in sports video tracking simulation system, verified the feasibility of the theory. In the analysis of the basic characteristics of sports video based on the proposed framework, mining basic motion features, and briefly introduces the function of each part of the framework, and the basic characteristics of the corresponding extraction technology. The basic features include: extracting trajectory extraction, extraction of moving object position, and the direction of movement of the extraction. This paper presents a hybrid tracking method mean shift algorithm and color histogram based algorithm, and introduces the principle of two kinds of algorithms. The hybrid algorithm can effectively avoid the interference problem of the moving object in complex background, greatly improve the detection accuracy of athletes. The MATLAB simulation results prove that the theory has an ideal effect.

\section{SURVEY OF TARGET DETECTION METHOD}

\subsection{Image Preprocessing Method}

Each pixel of the color image is usually represented by 3 bytes, each byte corresponds to the RGB component of the 
brightness, and a pixel gray level image transformed by a byte to represent, the little gray value greater brightness is bright; smaller values, brightness is black. Conversion between the commonly used formula [7]:

Grey $(i, j)=0.1 * r(i, j)+0.59 * g(z, j)+0.3 * b(i, j)$

The input value of gray level higher than the compressed into narrow output image in lighter gray level, the output is high contrast images:

$\lambda(m)=\frac{E\left(X(n) X(n+m)^{<p-1>}\right)}{E\left(|X(n+m)|^{p}\right)}$

According to the formula, Histogram equalization $(\mathrm{x}, \mathrm{x})$ on the input gray level executive changes the output gray level S.

$\hat{\lambda}(m)=\frac{\sum_{m=1}^{N} X(n)|X(n+m)|^{p-1} \operatorname{sign}[X(n+m)]}{\sum_{m=1}^{N}|X(n+m)|^{p}}$,

$1 \leq p<\alpha \leq 2$

In the formula (W) representation of the probability density function of the gray level in a given image, $\mathrm{W}$ is the 11 sub variable integral. You can get the probability density function of the output gray level is homogeneous:

$p_{s}(s)=\left\{\begin{array}{l}1,0 \leq s \leq 1 \\ 0, \quad \text { else }\end{array}\right.$

Image filtering is the most commonly used method of elimination image noise, common noise with salt and pepper noise, impulse noise, Gauss noise. In order to eliminate the noise or highlight some features of image, on the need for smoothing or sharpening images. Commonly used methods of median filter, mean filter, Gauss filter [8].

\subsection{Filter Processing}

Image filtering is the most commonly used method of elimination image noise, common noise with salt and pepper noise, impulse noise, Gauss noise. In order to eliminate the noise or highlight some features of image, on the need for smoothing or sharpening images [9-12]. Commonly used methods of median filter, mean filter, Gauss filter.

The filtering process is the first two-dimensional sliding template selection of specific structure, and then will be arranged according to the size of pixel values in the form template, the data sequence monotone. The output formula of two dimensional median for:

$G(x, y)=\operatorname{Mid} f(x-i, y-j),(i, j)$

Gaussian smoothing filter is a widely used algorithm in image processing, mathematical expressions for Gaussian smoothing filter:

$f^{\prime}(x, y)=f(x, y) * g(x, y)$ $g(x, y)=\frac{1}{\sqrt{2 \pi \sigma}} \exp \left(-\frac{x^{2}+y^{2}}{2 \sigma^{2}}\right)$

\subsection{Mathematical Morphology Processing}

Methods of mathematical morphology for image processing based on the geometry, it focuses on the research of image collection structure, this structure can be expressed on the macroscopic properties of objects, can also be said of the microscopic properties, structural elements and its basic idea is to use a certain structure and characteristics to measure image morphology. By using the basic operations of mathematical morphology, image processing, in order to improve the image effect.

Let $\mathrm{X}$ and $\mathrm{B}$ is the set of integer space in the $\mathrm{Z}$, where Ais the original image, and the B structure element.

a) Bon the expansion of Aoperations is defined as:

$A \oplus B=\left\{z \mid(\widehat{B})_{z} \cap A \neq \varnothing\right\}$

b) Corrosion of $\mathrm{B}$ on $\mathrm{A}$ to define operations:

$A \circ B=(A \Theta B) \oplus B$

c) $\mathrm{B}$ on $\mathrm{A}$ open operation is defined as:

$A \Theta B=\left\{z \mid(B)_{z} \subseteq A\right\}$

\subsection{Moving Target Detection Methods}

Moving object detection in image sequence is a difficult research field, and is very important in general, target detection in sports video is mainly to identify and analyze video moving object in the stream, filter out image and moving target irrelevant information, the moving object is separated from the scene.

Three towel character difference method the mathematical expression formula is as follows:

$$
\begin{aligned}
& m_{i}(i, j)=\left|f_{i-1}(i, j)-f_{i}(i, j)\right| \times\left|f_{i}(i, j)-f_{i+1}(i, j)\right| \\
& M_{t}(i, j)=\left\{\begin{array}{l}
255, m_{i}(i, j)>T \\
0
\end{array}\right.
\end{aligned}
$$

\subsection{Kalman Filter}

In the target tracking process, because the two towel character image is a short period of time, the state of the target changes very little, it can be assumed that the target in a time interval keeps uniform motion, so the movement velocity as a parameter to reflect the target.

Because the goal of hook speed in unit time interval, so the definition of state transition matrix:

$$
A=\left(\begin{array}{cccc}
1 & 0 & \Delta t & 0 \\
0 & 1 & 0 & \Delta t \\
0 & 0 & 1 & 0 \\
0 & 0 & 0 & 1
\end{array}\right)
$$


Table 1. SVMM compared with the traditional still exists a certain difference.

\begin{tabular}{|c|c|c|c|c|}
\hline Type & Feature Type & Contains Range & Objective & Mining Method \\
\hline Multimedia mining & $\begin{array}{l}\text { Text, audio, visual, motion, } \\
\text { multimodal features fusion }\end{array}$ & All media files & $\begin{array}{l}\text { Query, the acquisition of } \\
\text { knowledge }\end{array}$ & $\begin{array}{c}\text { Statistics, clustering, Asso- } \\
\text { ciation }\end{array}$ \\
\hline Video mining & $\begin{array}{l}\text { Color, texture, shape, mo- } \\
\text { tion, semantic features }\end{array}$ & Video files & $\begin{array}{l}\text { Query, the acquisition of } \\
\text { knowledge }\end{array}$ & $\begin{array}{c}\text { Statistics, clustering, Asso- } \\
\text { ciation } \\
\text { SVMM }\end{array}$ \\
\hline SVMM & Motion characteristics & Sports video files & $\begin{array}{c}\text { Query, knowledge acquisi- } \\
\text { tion }\end{array}$ & $\begin{array}{c}\text { Statistics, clustering, Asso- } \\
\text { ciation }\end{array}$ \\
\hline
\end{tabular}

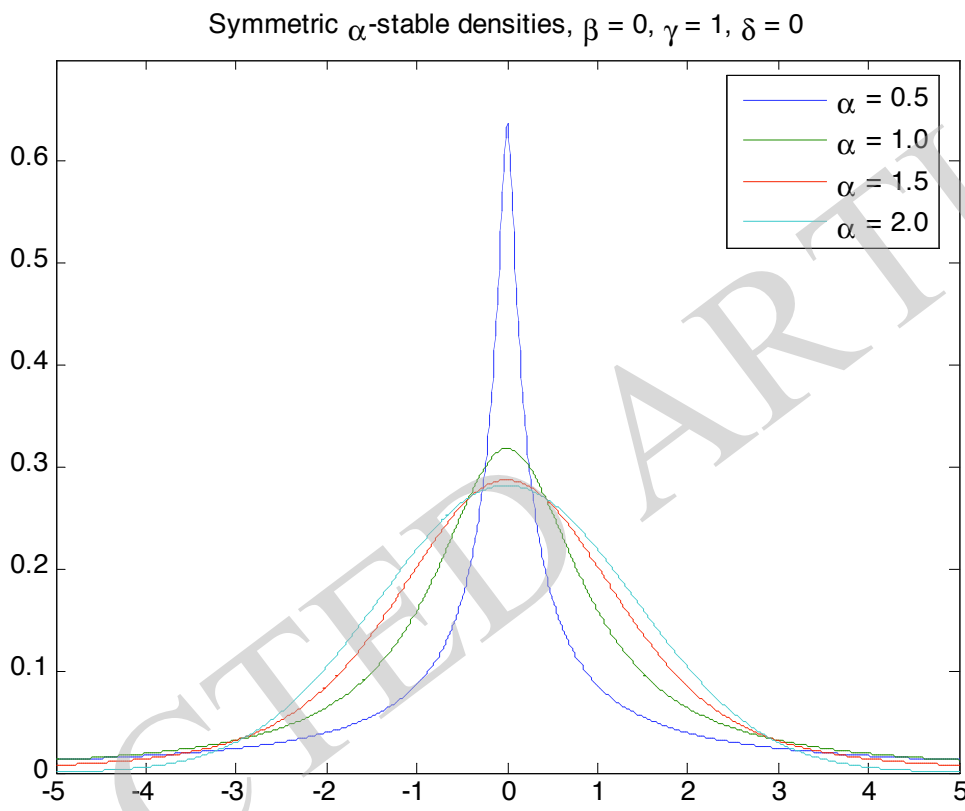

Fig. (1). Three levels of three levels and in multimedia data mining.

Type in $\Delta \mathrm{T}$ and time $\mathrm{T}-1$ said time interval, the system state and the observation state know, observation matrix:

$$
H=\left(\begin{array}{llll}
1 & 0 & 0 & 0 \\
0 & 1 & 0 & 0
\end{array}\right)
$$

\section{FRAME THEORY LEVEL AND THE SYSTEM OF SPORTS VIDEO MOTION MINING}

\subsection{The Basic Concept}

Sports video mining research scholars in the area of relatively small, content is relatively single, mainly focus on the research content is either mining content based video, or is the extraction of motion features, so, must have the thorough research to it, must be some basic concepts are clear.

Sports video mining to extract the relevant data from the database or data warehouse, the motion analysis for motion characteristics, using certain data mining method, get the hidden patterns or knowledge. The motion features in SVMM can be the basic features of the motion characteristics of movement, which does not contain semantic informa- tion, can also be the motion characteristics include semantic, though the two are movement characteristics, but their effect on the extraction of implicit knowledge is not the same. Of course, in reality, motion features not containing semantic information does not exist. Multimedia mining, video mining SVMM compared with the traditional still exists a certain difference, the comparison are given in Table $\mathbf{1}$.

\subsection{The Theoretical Level}

According to the analysis of SVMM, this paper will be divided into the following three levels: basic level, model, event layer and knowledge layer. Three levels of three levels and in multimedia data mining is the opposite, as shown in Fig. (1), the basic characteristics of layer corresponding to the lower layer, model, event layer corresponding to the middle layer, knowledge layer corresponding to the semantic layer.

\subsection{Hybrid Tracking Algorithm}

Model, event layer contains event detection technology, through the event detection, model, we can get some basic, 


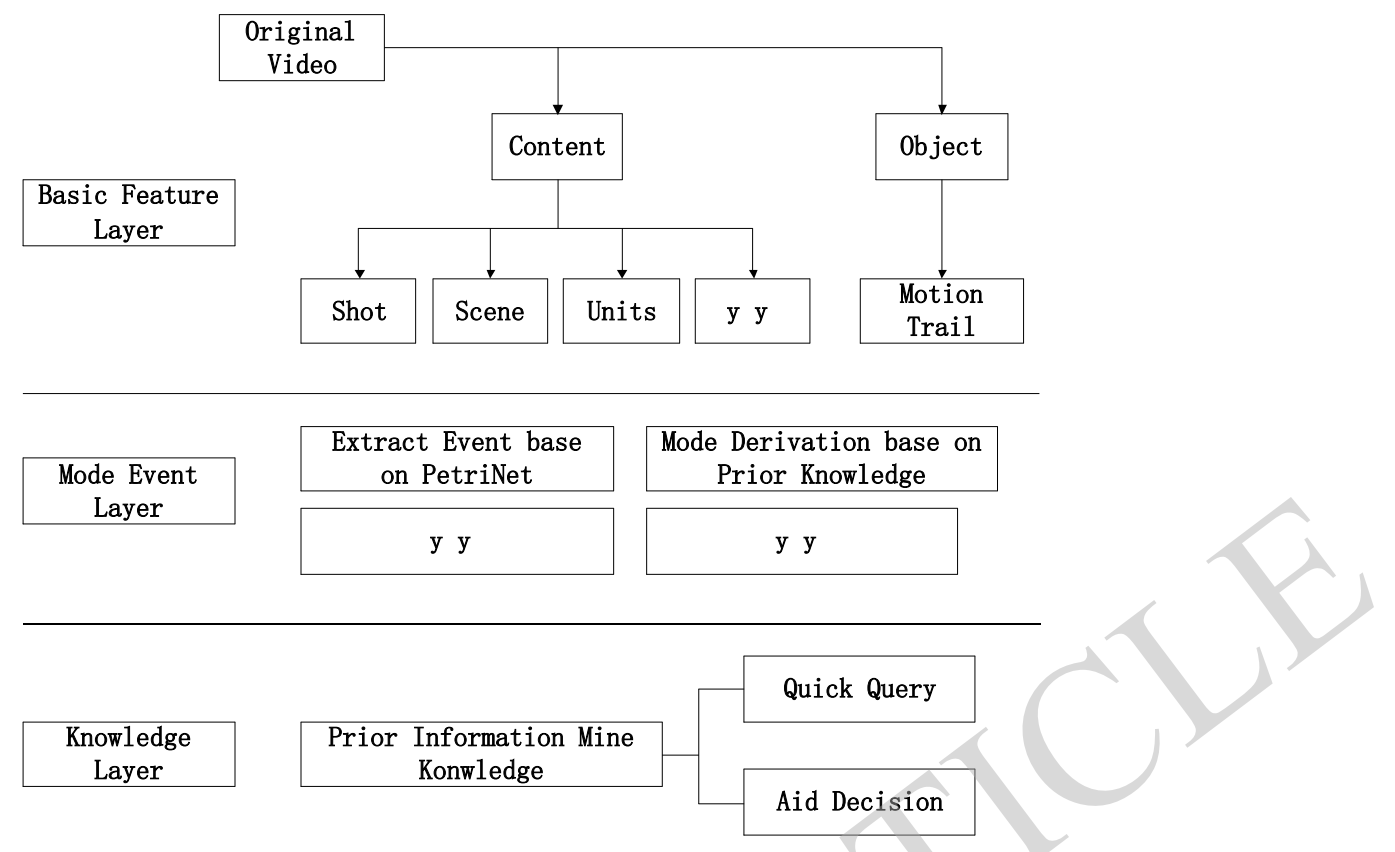

Fig. (2). The specific technical route.

primitive knowledge; in order to obtain some advanced, implicit knowledge, must be obtained after analysis, at the same time, obtain knowledge, these knowledge and feedback, can to provide users with inquiry and assistant decision-making function. The specific technical route was shown in Fig. (2).

\section{RESULTS AND SIMULATION}

\subsection{Mining Basic Motion Characteristics}

The motion characteristics of mining, the main steps for design reference system framework, the main process was shown in Fig. (3).

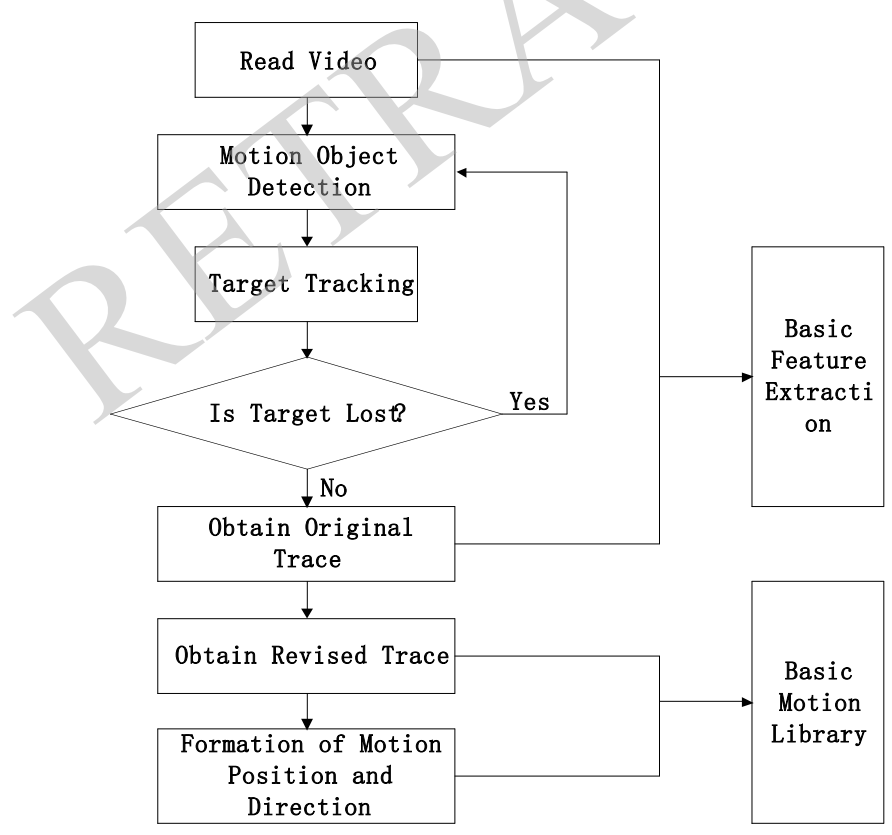

Fig. (3). The main motion process characteristics of mining.

\subsection{Detection of Moving Objects}

Step one: target tracking initialization of the model of the current frame.

Step two: the candidate target window initialization of a new meal in the image. The candidate target initialization process, the initial target center position and the target pixel, and according to the formula (12), (13), (14) the shape and color feature of initial candidate targets are calculated.

Step three: Bhattacharyya coefficient $\mathrm{P}$ calculated the candidate target andtarget model.

Step four: if according to the mean shift algorithm to update the candidate targetposition according to a shape, V color histogram algorithm to update the candidate target.

Step five: repeat step two and step three, until the $\mathrm{Pi}<\mathrm{Pi}$ 1. Finally get to meet the new position of the Bhattacharyya coefficient to maximize the target tracking.

This paper verified to the effect of the algorithm, in the MATLAB platform for thegame of golf video tracking simulation experiment. Tracking results of golf video game was shown in Fig. (4).

\subsection{Extraction Correcting Trajectory}

Through the detection and moving object tracking of the object, can the original trajectory of the object is obtained, in order to get the trajectory and motion direction effectively, it must first be original corrected trajectory, trajectory correction. As shown in Fig. (5), the moving object is a video sequence of the trajectory, it is difficult to reflect the real motion of moving objects.

$\frac{\mathrm{L} 2}{12}=\frac{\sqrt{(\mathrm{d}+\mathrm{d} 1)^{2}+\mathrm{h}^{2}}}{\mathrm{f}}$ 

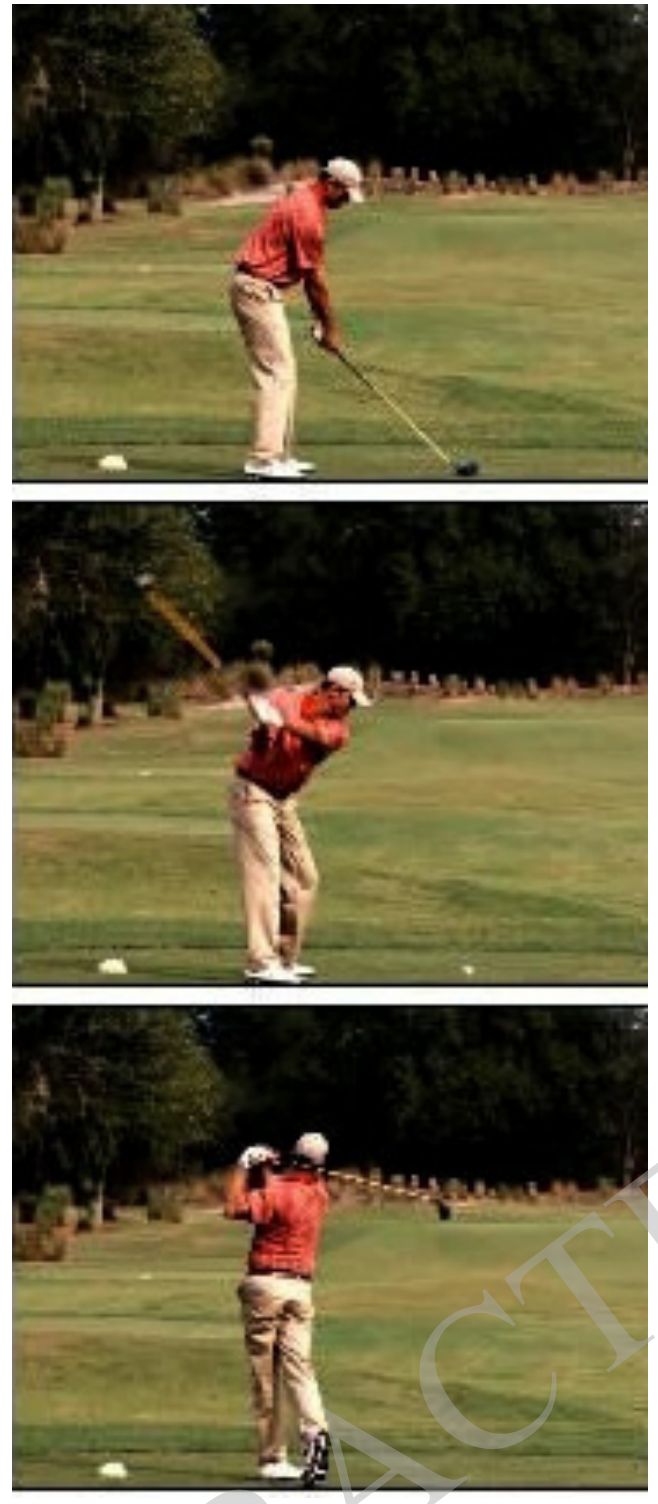

Fig. (4). Tracking results of golf video game.

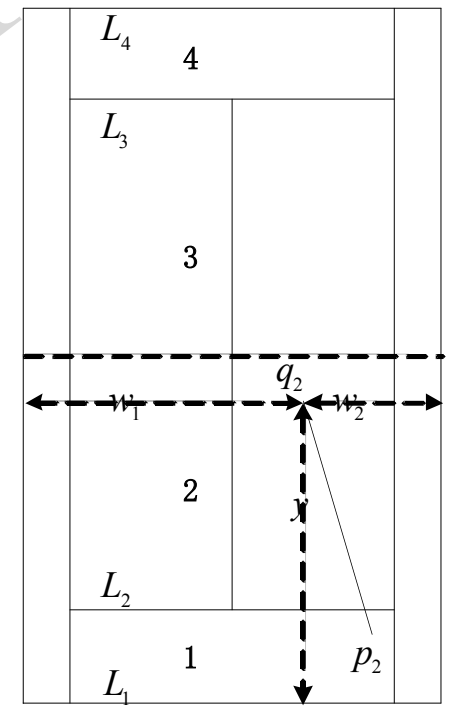

$$
\begin{aligned}
& \frac{L 3}{13}=\frac{\sqrt{(d+2 H-d 3)^{2}+h^{2}}}{f} \\
& \frac{L 4}{14}=\frac{\sqrt{(d+2 H)^{2}+h^{2}}}{f}
\end{aligned}
$$

\subsection{Experimental Results and Analysis}

SVMMP mainly consists of extraction is the basic characteristic, mode, event, knowledge mining (MEK mining) and query of three parts. Among them, the basic feature extraction is based on MEK mining, MEK mining is the basic purpose of feature extraction and representation, both of which can provide the query service, query function reflects the hierarchical characteristics of sports video mining, can meet the needs of different users. Fig. (6) shows the design idea of SVMMP.

Color information is compared with other feature information of target rotation, translation, deformation, occlusion is not sensitive, and compared with otherfeature information more convenient, is widely used in image processing, image segmentation, target detection and tracking in computer vision. In a video image, color distribution was shown in Fig. (7).

\section{CONCLUSION}

In this paper, the sports video was the research object, to a certain extent, the method of sports video mining. Sports video mining hierarchical structure is a Pyramid shaped: the basic feature of layer, model, event layer and knowledge layer, which corresponds to the multimedia data mining in the low level features, mid-level features and high-level semantic mining. On the base of sports video motion features in-depth analysis, proposed the motion feature includes a motion track, direction and a plurality of moving target relative position, this paper mainly studies the movement of the position, direction of motion of two aspects. The basic steps in the basic characteristics of motion mining first briefly

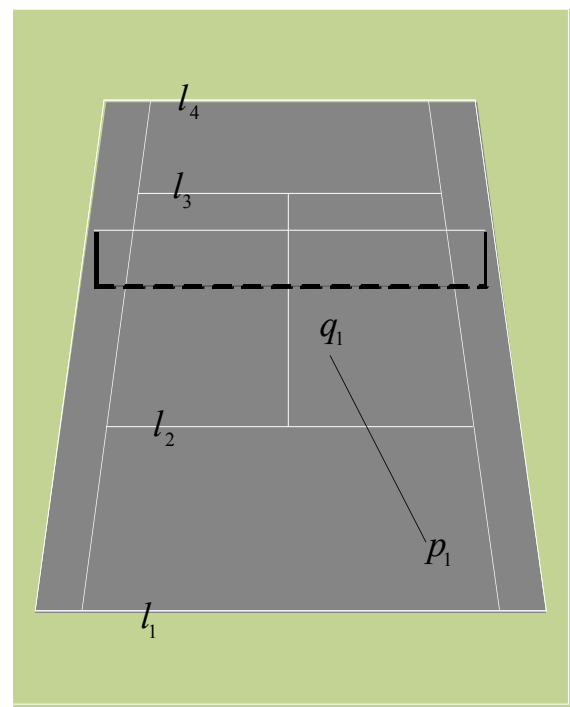

Fig. (5). Real court and changed court. 


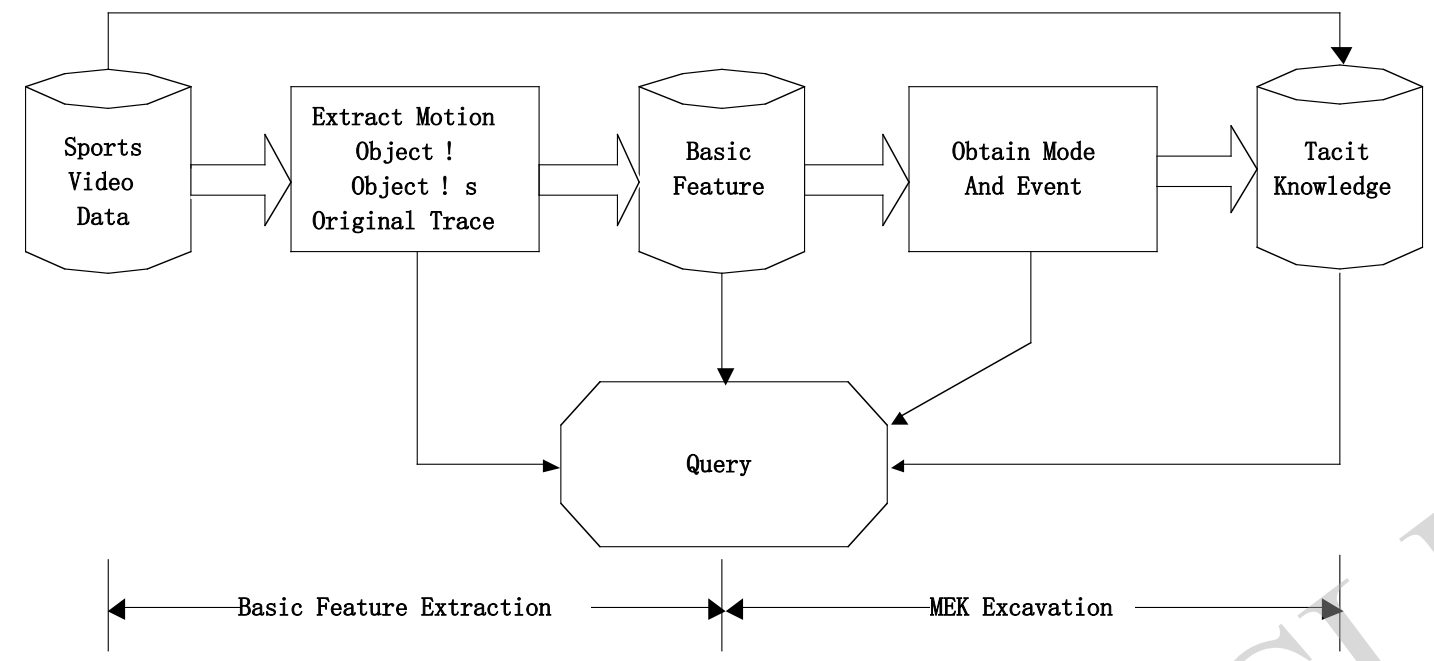

Fig. (6). The design idea of SVMMP.
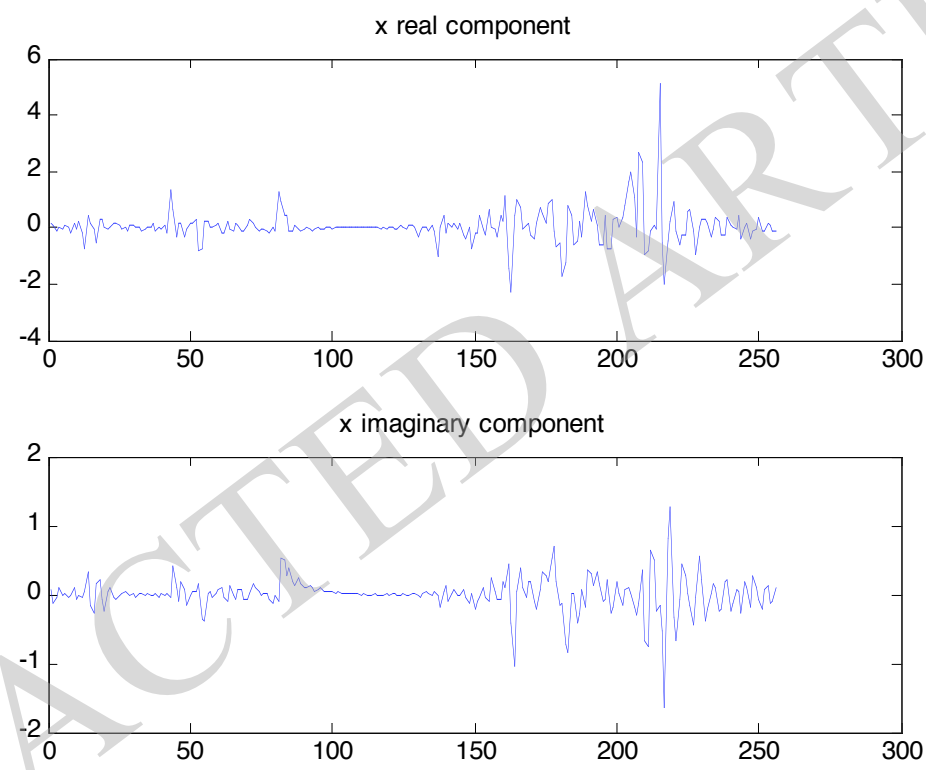

Fig. (7). Color distribution in a video image.

introduces the basic sports video feature mining method; then introduces the detection and moving objects tracking moving objects in SVMM; finally, mainly introduces the correct trajectory.

\section{CONFLICT OF INTEREST}

The authors confirm that this article content has no conflict of interest.

\section{ACKNOWLEDGEMENTS}

Declared none.

\section{REFERENCES}

[1] H. Li, L. Guo, and H. Liu, "Research on image fusion based on the two generation Curvelet transform", Journal of applied optics, vol. 8, no. 2, pp. 143-156, 2007.

[2] W. Zhao, and S. Mao, "A pixel-level multisensor image fusion algorithm based on false color", Chinese Journal of Electronics, vol. 3, no. 31, pp. 368-371, 2003.

[3] D. Abbott, S. Cunningham, G. Daniels, B. Doyle, J. Dumlop, D Economo, T. Farmer, D. Farrant, C. Foley, B. Fox, M. Hedley, J. Herrmann, and C. Jacka, "Development and Evaluation of Sensor Concepts for Ageless Aerospace Vehicles - Threats and Measurands," NASA, vol. 2, no. 11, pp. 68-77, 2002.

[4] J. Z. Li, J. Li, and S. F. Shi, "Concepts, Issues and Advance of Sensor Networks and Data Management of Sensor Networks," Journal of Software, vol. 14, no. 10, pp. 1717-1727, 2003.

[5] Tilak S, Abu-Ghazaleh NB, Heinzelman W, "A Taxonomy of Wireless Micro-sensor Network models," Mobile Computing and Commuications Review, vo. 1, no. 2, pp. 1-8, 2002.

[6] David Culler, Deborah Estrin, Mani Srivastava, "Overview of Sensor Networks", IEEE Computer Society Officers, vol. 11, no. 10, pp. 15-25, 2005.

[7] T. Matsuo, Y. Kaneko, M. Matano, "Introduction of Intelligent Vehicle Detection Sensors", IEEE/IEEJ/JSAI International Conference on Intelligent Transportation Systems, vol. 5, no. 12, pp. 709713, 1999.

[8] J. Deng, R. Han, S. Mishra, "Inrusion tolerance and Anti-traffic Analysis Strategies in Wireless Sensor Networks," IEEE 2004 International Conference on Dependable Systems and Networks, vol. 10, no. 11, pp. 477-490, 2004. 
[9] Q.R. Li, L.Y. Wei, and S.F. Ma, "The Model Analysis of Vehicles Situation and Distribution in Intersections Abased on Markov Process," IEEE International Conference on Intelligent Transportation Systems, vol. 2, no. 10, pp. 1076-1080, 2003.

[10] A. Wadaa, S. Olariu, L. Wilson, M. Eltoweissy, and K. Jones, "On Providing Anonymity in Wireless Sensor Networks," 10th International Conference on Parallet and Distributed Systems, vol. 5, no 10, pp. 125-136, 2004.
[11] S. Wang, and X. Zhu, “ $\alpha$ spectrum estimation method for ARMA $S \alpha S$ process based on FLOC," Journal on Communications, vol. 28, no. 7, pp. 98-103, 2007.

[12] H. B. Wang, J. B. Long, and D. F. Zha, "Pseudo Cohen TimeFrequency Distributions in Infinite Variance Noise Environment," Applied Mechanics and Materials, vol. 475-476, pp. 253-258, 2013.

Received: June 10,2015

Revised: July 29, 2015

Accepted: August 15,2015

(C) Tan and Kang; Licensee Bentham Open.

This is an open access article licensed under the terms of the (https://creativecommons.org/licenses/by/4.0/legalcode), which permits unrestricted, noncommercial use, distribution and reproduction in any medium, provided the work is properly cited. 